\title{
A novel aldo-keto reductase gene, IbAKR, from sweet potato confers higher tolerance to cadmium stress in tobacco
}

\author{
Jinxi HUO, Bing DU, Sifan SUN, Shaozhen HE, Ning ZHAO, Qingchang LIU, Hong ZHAI (凶)
}

Key Laboratory of Sweetpotato Biology and Biotechnology, Ministry of Agriculture/Beijing Key Laboratory of Crop Genetic Improvement/ Laboratory of Crop Heterosis and Utilization, Ministry of Education, China Agricultural University, Beijing 100193, China

\begin{abstract}
High concentrations of $\mathrm{Cd}$ can inhibit growth and reduce the activity of the photosynthetic apparatus in plants. In several plant species, aldo-keto reductases (AKRs) have been shown to enhance tolerance to various abiotic stresses by scavenging cytotoxic aldehydes; however, few AKRs have been reported to enhance $\mathrm{Cd}$ stress tolerance. In this study, the gene $I b A K R$ was isolated from sweet potato. The relative expression levels of $I b A K R$ increased significantly (approximately 3 -fold) after exposure to $200 \mu \mathrm{mol} \cdot \mathrm{L}^{-1} \mathrm{CdCl}_{2}$ or $10 \mathrm{mmol} \cdot \mathrm{L}^{-1} \mathrm{H}_{2} \mathrm{O}_{2}$. A subcellular localization assay showed that IbAKR is predominantly located in the nucleus and cytoplasm. $I b A K R$-overexpressing tobacco plants showed higher tolerance to Cd stress than wild-type (WT). Transgenic lines showed a significant ability to scavenge malondialdehyde (MDA) and methylglyoxal (MG). In addition, proline content and superoxide dismutase activity were significantly higher and $\mathrm{H}_{2} \mathrm{O}_{2}$ levels were significantly lower in the transgenic plants than in the WT. Quantitative real-time PCR analysis showed that the reactive oxygen species (ROS) scavenging genes encoding guaiacol peroxidase $(G P X)$, ascorbate peroxidase $(A P X)$, monodehydroascorbate reductase $(M D H A R)$ and peroxidase $(P O D)$ were significantly upregulated in transgenic plants compared to WT under Cd stress. These findings suggest that overexpressing $I b A K R$ enhances tolerance to Cd stress via the scavenging of cytotoxic aldehydes and the activation of the ROS scavenging system.
\end{abstract}

Keywords cadmium stress, IbAKR, Ipomoea batatas, sweet potato

\section{Introduction}

The heavy metal, $\mathrm{Cd}$, is considered to be biologically

Received March 7, 2018; accepted April 8, 2018

Correspondence: zhaihong@cau.edu.cn nonessential for almost all plants. With rapid industrialization and the widespread use of agrochemicals in agriculture in recent years, very large areas of land have been polluted by $\mathrm{Cd}$. In China, about $7.75 \%$ of farmland soil is polluted by $\mathrm{Cd}^{[1]}$. The effect of Cd toxicity on plants includes the inhibition of growth and a reduction in the activity of the photosynthetic apparatus ${ }^{[2]}$. These situations pose a continuously increasing burden to global crop productivity, and hence, there is demand for crop varieties that are adaptive and resistant to various stresses. Genetic engineering has the potential to improve tolerance to $\mathrm{Cd}$ stress in plants ${ }^{[3]}$.

The aldo-keto reductase (AKR) protein superfamily contains $>190$ members and is found in all phyla. The AKR superfamily comprises 18 families and includes mainly $\mathrm{NAD}(\mathrm{P})(\mathrm{H})$-dependent oxidoreductases. These enzymes reduce carbonyl substrates, such as lipid peroxidation byproducts, keto-steroids, keto-prostaglandins, retinals and quinones ${ }^{[4]}$. In plants, AKRs have been reported to enhance tolerance to various abiotic stresses. The overexpression of a novel alfalfa aldose/aldehyde reductase gene in transgenic tobacco plants enhanced tolerance to oxidative damage caused by paraquat, heavy metals, UV-B (280-320 nm) irradiation, drought and low temperature ${ }^{[5-7]}$. The overexpression of a rice $A K R$ increased tolerance to oxidative stress, heat and glyphosate in transgenic rice ${ }^{[8,9]}$. An aldose reductase from Xerophyta viscosa conferred enhanced drought and salinity tolerance in transgenic tobacco plants ${ }^{[10]}$. The expression of an Arabidopsis thaliana-encoded AKR protein in transgenic barley increased tolerance to oxidative stress, Cd stress and freezing stress ${ }^{[11,12]}$. Salt tolerance increased in Arabidopsis transformed with $P p A K R 1$, which had been cloned from peach (Prunus persica) ${ }^{[13]}$. Recently, a novel member of the AKR superfamily was cloned from a soil Sphingomonas strain and was responsible for detoxifying the Fusarium toxin deoxynivalenol ${ }^{[14]}$. Regenerated transgenic tobacco and rice lines expressing PsAKR1, an $A K R$ gene from Pseudomonas, showed improved tolerance to accelerated aging and glyphosate ${ }^{[9,15]}$. However, the role 
of AKRs in Cd stress tolerance has rarely been reported.

Sweet potato, Ipomoea batatas, is an important root crop, and the storage root is widely used in the energy industry ${ }^{[16]}$. In addition, the concentrations of $\mathrm{Cd}$ is lower in the storage root tissue than in the fibrous root, stem and leaves grown in Cd-contaminated farmland ${ }^{[17]}$, suggesting that sweet potato, as a source of bioenergy, could be a candidate crop for cultivation in Cd-contaminated soil. Therefore, it is of great significance to explore the $\mathrm{Cd}$ stress-responsive genes in sweet potato to improve its $\mathrm{Cd}$ tolerance. In this study, a novel aldo/keto reductase gene, $I b A K R$, was cloned from sweet potato cv. Lushu 3 , and it was found that the overexpression of IbAKR resulted in increased tolerance to $\mathrm{Cd}$ stress in transgenic tobacco plants.

\section{Materials and methods}

\subsection{Plant materials}

The sweet potato cv. Lushu 3 was used for $I b A K R$ gene cloning and expression analysis. Tobacco (Nicotiana tabacum) cv. Wisconsin 38 was used to study the functions of $I b A K R$.

2.2 Cloning and sequence analysis of the sweet potato IbAKR gene

TransZol UP (TransGen Biotech, Beijing, China) was used to extract total RNA from fresh leaves of 4-week-old in vitro-grown Lushu 3 plants. To amplify the full-length IbAKR sequence, rapid amplification of cDNA ends (RACE) was performed using a $5^{\prime} / 3^{\prime}$ full RACE kit (Takara, Dalian, China). Gene-specific primers (5GSP1 and 5GSP1-2/3GSP1 and 3GSP1-2) were used for 5' and $3^{\prime}$ RACE. The open reading frame of $I b A K R$ was predicted using ORF finder. The conserved domains of the IbAKR protein were analyzed using NCBI BLAST. For the multiple sequence alignment analysis, the amino acid sequences of IbAKR and other AKR members were retrieved from the AKR superfamily home page and aligned using ClustalW. Phylogenetic analysis was conducted using MEGA6 software. The theoretical molecular weight and isoelectronic point $(p \mathrm{I})$ were calculated at ProtParam. The primers mentioned above are listed in supplementary Table S1.

\subsection{IbAKR expression analysis}

Total RNA was extracted from different tissues as described above. About $1 \mu \mathrm{g}$ of total RNA was reverse transcribed using a PrimeScript RT reagent kit with gDNA Eraser (Takara, Dalian, China) according to the manufacturer's protocol. The cDNA solution was used as a template for PCR amplification. The sweet potato Ibactin gene was used as an internal control. Quantitative real-time PCR (qRT-PCR) was carried out in a $10 \mu \mathrm{L}$ volume using SYBR Premix Ex Taq (Takara, Dalian, China) and a 7500 Real-Time PCR system (Applied Biosystems, Foster City, CA, USA). Gene expression was quantified using the comparative $C_{\mathrm{T}}$ method $^{[18]}$. The primers mentioned above are listed in Table S1.

\subsection{Subcellular localization assay}

The full-length $I b A K R$ coding region without the termination codon was inserted into the pMDC83-GFP vector via PCR with linker primers (pMDC83-AKR-F/R) that contained $P a c \mathrm{I}$ and $A s c \mathrm{I}$ sites, generating a C-terminal fusion with the GFP gene under the control of the CaMV $35 \mathrm{~S}$ promoter. All plasmid constructs were confirmed by sequencing. The vector 35S:GFP, which served as the control, and 35S:IbAKR-GFP were transiently expressed in onion epidermal cells using a biolistic particle delivery system (PDS-1000 Bio-Rad, Hercules, CA, USA). The subcellular localization of the IbAKR-GFP protein was observed with a confocal laser scanning microscope under $488 \mathrm{~nm}$ excitation (EZ-C1, Nikon, Tokyo, Japan). The primers mentioned above are listed in Table S1.

\subsection{Generation of transgenic plants}

The expression cassette $\mathrm{P}_{35 \mathrm{~S}}-I b A K R-\mathrm{T}_{\mathrm{NOS}}$ was inserted into the plant binary vector pCAMBIA3301 at the $X b a \mathrm{I}$ and $S a c$ I sites. The sequence-verified plasmid was transfected into Agrobacterium tumefaciens strain EHA105 using the freeze-thaw method. Tobacco leaf explants were used for transformation as described by Horsch et al. ${ }^{[19]}$. Both GUS staining and PCR analysis (Bar-F/R) were conducted to confirm positive transgenic plants. The primers mentioned above are listed in Table S1.

\subsection{Assay for cadmium tolerance}

An in vitro assay for $\mathrm{Cd}$ tolerance was conducted at $28^{\circ} \mathrm{C}$ using $1 / 2 \mathrm{MS}$ medium with $200 \mu \mathrm{mol} \cdot \mathrm{L}^{-1} \mathrm{CdCl}_{2}$ and a $16 /$ $8 \mathrm{~h} \mathrm{light/dark}$ cycle. Three plants per line were treated. The growth and rooting ability were continuously observed for 4 weeks. In the pot experiment, 8-week-old tobacco plants were planted in a transplanting box and irrigated with Hoagland solution with $100 \mathrm{mmol} \cdot \mathrm{L}^{-1} \mathrm{CdCl}_{2}$ every three days. Morphological changes in these lines were observed after 2 weeks.

2.7 Analyses of MDA, proline and $\mathrm{H}_{2} \mathrm{O}_{2}$ content and SOD activity

The malondialdehyde (MDA) content was measured according to the method of Gao et al. ${ }^{[20]}$. The proline content and superoxide dismutase (SOD) activity were analyzed as described by $\mathrm{He}$ et al. ${ }^{[21]} \cdot \mathrm{H}_{2} \mathrm{O}_{2}$ content was 
measured according to the method of Alexieva et al. ${ }^{[22]}$.

2.8 Leaf disc senescence assay and measurement of chlorophyll content

The leaf disc senescence assay was conducted according to Kumar et al. ${ }^{[10]}$. To measure the chlorophyll content, $0.2 \mathrm{~g}$ of leaf discs were ground in $80 \%$ buffered aqueous acetone. The homogenate was filtered through filter paper and diluted to $25 \mathrm{~mL}$ with $80 \%$ aqueous acetone. The chlorophyll $\mathrm{a}$ and $\mathrm{b}$ content of the solution was measured at 663 and $645 \mathrm{~nm}$, respectively, using a spectrophotometer. The chlorophyll concentration was calculated as described by Porra et al. ${ }^{[23]}$.

\subsection{Expression analysis of ROS scavenging-related genes}

Transgenic and wild-type (WT) plants were treated with $100 \mathrm{mmol} \cdot \mathrm{L}^{-1} \mathrm{CdCl}_{2}$ for 2 weeks, and the third leaves from the top were used to analyze the expression of reactive oxygen species (ROS) scavenging-related genes guaiacol peroxidase $(G P X)$, ascorbate peroxidase $(A P X)$, monodehydroascorbate reductase $(M D H A R)$ and peroxidase $(P O D)$. Tobacco Ntactin was used as an internal control, and qRT-PCR analysis was conducted as described above. The qRT-PCR primers are listed in Table S1.

\subsection{Statistical analysis}

The experiments were repeated three times, the data were analyzed using Student's two-tailed $t$-test, and the results are presented as the mean \pm SE. A value of $P<0.05$ or $<0.01$ was considered statistically significant.

\section{Results}

\subsection{Analysis of IbAKR characteristics}

The IbAKR gene was isolated from the sweet potato cultivar Lushu 3. It consisted of five exons and four introns and contained a $1053 \mathrm{bp}$ ORF that encoded a 350-aminoacid polypeptide with a calculated molecular weight of $38.32 \mathrm{kDa}$ and a $p \mathrm{I}$ of 5.85. BLAST showed that the AKR protein contained typical AKR signature motifs (Fig. S1a), including an $(\alpha / \beta)_{8}$-barrel motif and catalytic tetrad (Asp63, Tyr-68, Lys-94, and His-135), which are conserved in the AKR superfamily. The AKR superfamily comprises 18 families and more than 170 members (as of July 2017, families $16-18$ had not been included $)^{[4,14,24]}$. A phylogenetic analysis of IbAKR showed high amino acid identity with Rauvolfia serpentina AKR13D1 (AY766462, $51.63 \%$ ) (Fig. S1b). Based on the nomenclature criteria for the AKR family ${ }^{[25]}$, IbAKR is in family 13 of the AKR superfamily.

\subsection{Analysis of $I b A K R$ expression}

qRT-PCR analysis showed $I b A K R$ expression in all organs of sweet potato examined. It was highly expressed in the roots of 4-week-old in vitro-grown Lushu 3 plants (Fig. 1a). After 4 weeks growth in vitro Lushu 3 plants were treated with $200 \mu \mathrm{mol} \cdot \mathrm{L}^{-1} \mathrm{CdCl}_{2}$ and $10 \mathrm{mmol} \cdot \mathrm{L}^{-1}$ $\mathrm{H}_{2} \mathrm{O}_{2}$ for $0,3,6,12,24$ and $48 \mathrm{~h}$. An increase in $I b A K R$ transcripts was observed after exposure to $200 \mu \mathrm{M} \mathrm{CdCl}_{2}$ and peaked at $6 \mathrm{~h}$ when the expression level was 3 times higher than that at $0 \mathrm{~h}$ (Fig. 1b). Under $10 \mathrm{mmol} \cdot \mathrm{L}^{-1} \mathrm{H}_{2} \mathrm{O}_{2}$ stress, the expression of $I b A K R$ was induced to a higher level (3.5-fold increase) at $12 \mathrm{~h}$ (Fig. 1c).

\subsection{Subcellular localization of IbAKR}

The subcellular localization of the IbAKR protein was investigated by generating $35 \mathrm{~S}_{\mathrm{pro}}-I b A K R-G F P$ and transiently expressing it in onion epidermal cells. The empty vector $35 \mathrm{~S}_{\text {pro- }}-G F P$ was used as a control. As shown in Fig. 2, the GFP fluorescence signal of IbAKR-GFP was detected in the nucleus and cytoplasm, as was the GFP fluorescence signal of the GFP control.
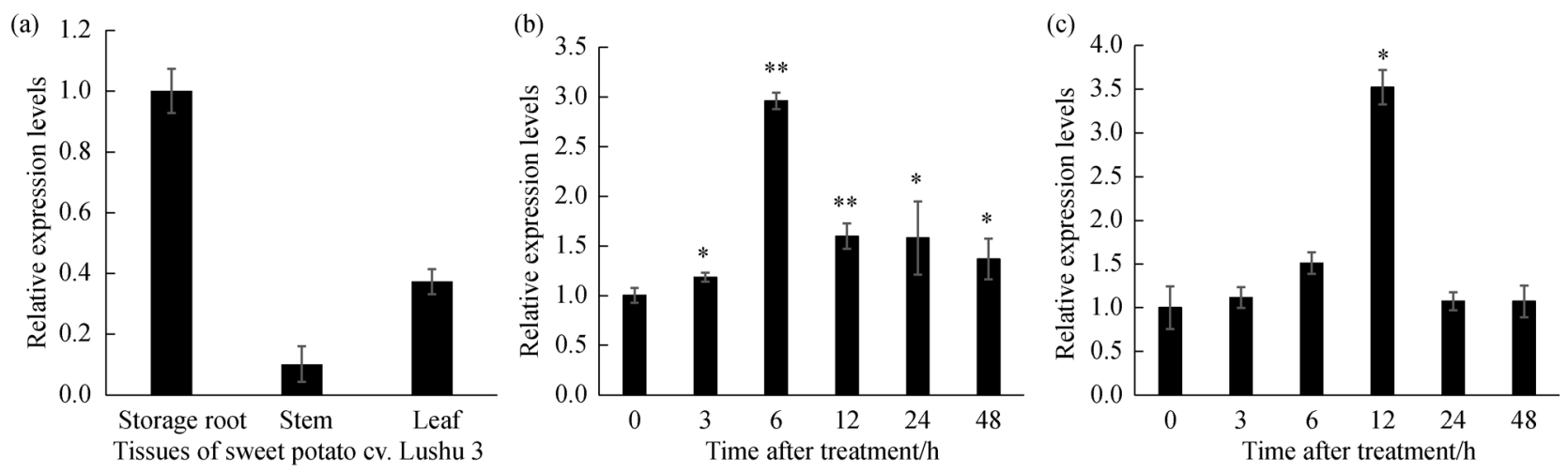

Fig. 1 Analysis of $I b A K R$ expression. (a) Organ-specific expression assay of $I b A K R$; (b,c) expression levels of $I b A K R$ after treatment with $200 \mu \mathrm{mol} \cdot \mathrm{L}^{-1} \mathrm{CdCl}_{2}$ (b) or $10 \mathrm{mmol} \cdot \mathrm{L}^{-1} \mathrm{H}_{2} \mathrm{O}_{2}$ (c). Data are presented as the mean $\pm \mathrm{SE}(n=3)$ * and ** indicate a significant difference from $0 \mathrm{~h}$ at $P<0.05$ and $<0.01$, respectively, by Student's $t$-test. 

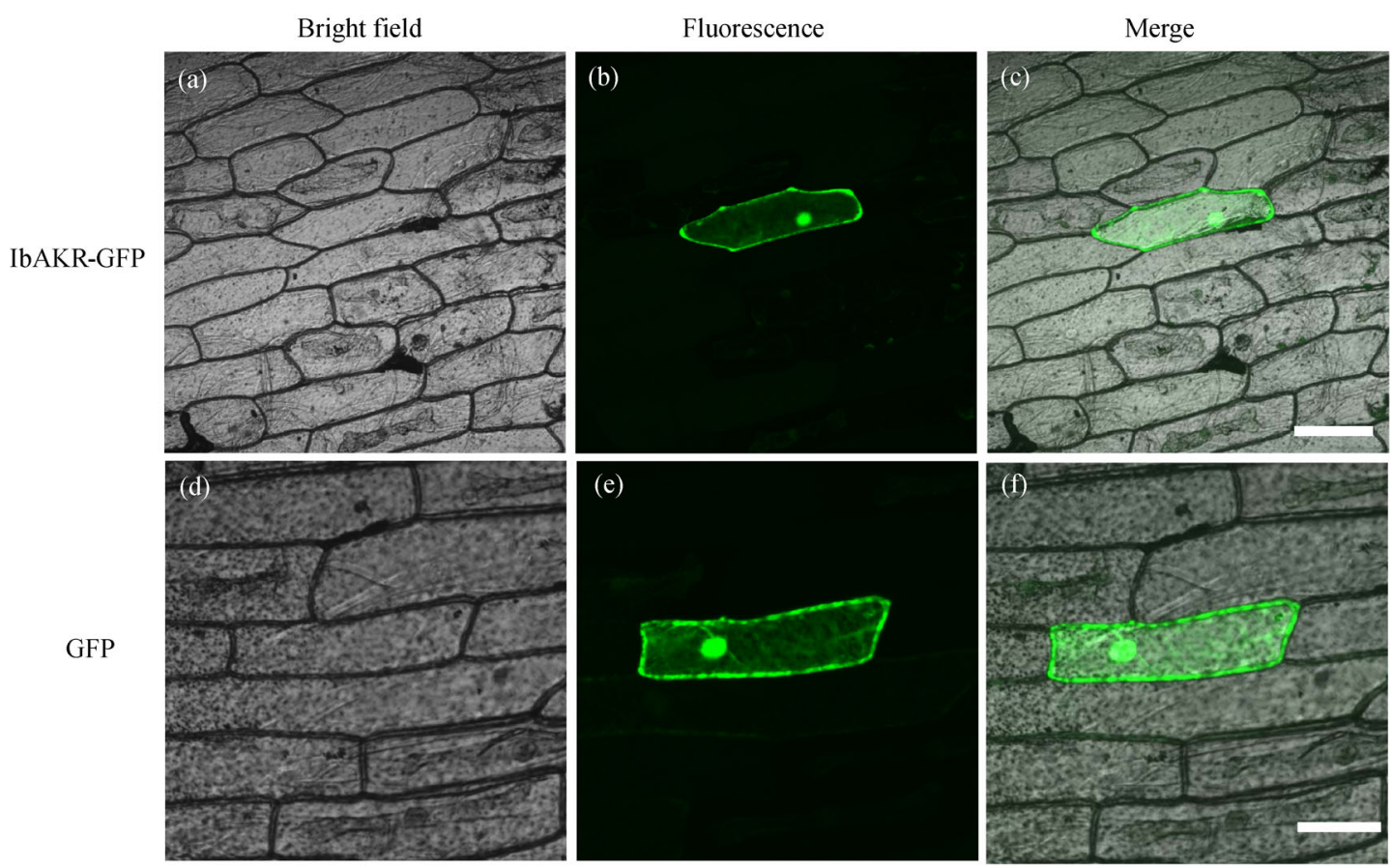

Fig. 2 Subcellular localization of the IbAKR-GFP fusion protein in onion epidermal cells. The empty 35S:GFP vector (GFP) (as a control) and 35S:IbAKR-GFP (IbAKR-GFP) were transiently expressed in onion epidermal cells. Fluorescence from AKR-GFP and GFP were observed using a confocal laser scanning microscope under $488 \mathrm{~nm}$ excitation. Bar $=100 \mu \mathrm{m}$.

\subsection{Overexpression of $I b A K R$ conferred strong cadmium stress tolerance to transgenic tobacco plants}

To elucidate the function of $I b A K R$ in plants, six transgenic tobacco lines overexpressing $I b A K R$ were generated (L1L6). The expression level of $I b A K R$ was significantly higher in the transgenic plants than in the WT plants. Two lines (L1, L2) were selected for further functional analysis.

To examine the role of $I b A K R$ in Cd stress, the WT and transgenic plants were cultured on $1 / 2 \mathrm{MS}$ medium with 0 or $100 \mu \mathrm{mol} \cdot \mathrm{L}^{-1} \mathrm{CdCl}_{2}$ for 4 weeks. The growth and rooting of all plants were normal without $\mathrm{CdCl}_{2}$. With $100 \mu \mathrm{mol} \cdot \mathrm{L}^{-1} \mathrm{CdCl}_{2}$, the growth and rooting development of both the WT and transgenic plants were restrained, but the Cd tolerance was significantly higher in the transgenic plants than in the WT (Fig. 3a-3c). To further test the role of $I b A K R$ in Cd stress tolerance, the transgenic plants and WT were grown in a transplanting box and irrigated with a $200 \mathrm{~mL}$ of $100 \mathrm{mmol} \cdot \mathrm{L}^{-1} \mathrm{CdCl}_{2}$ every $2 \mathrm{~d}$. After 2 weeks, compared to the two transgenic lines, the WT showed more severe chlorosis and necrosis (indicated by red circle in Fig. 4a). The transgenic plants showed significantly lower contents of MDA and $\mathrm{H}_{2} \mathrm{O}_{2}$ and significantly higher contents of proline than WT (Fig. 4b-4e).

\subsection{Overexpression of $I b A K R$ reduced the toxic effects of MG}

The levels of methylglyoxal (MG), a cytotoxic compound, increase upon exposing plants to various abiotic stresses. High concentrations of MG are very harmful to plants. The responses of the transgenic lines to MG stress were tested using detached leaf disc senescence assays. Leaf discs from WT and L2 were floated on different concentrations of MG for $2 \mathrm{~d}$. As shown in Fig. 5, the chlorophyll contents of WT and L2 showed no difference with 0 and $5 \mathrm{mmol} \cdot \mathrm{L}^{-1} \mathrm{MG}$; however, with $10 \mathrm{mmol} \cdot \mathrm{L}^{-1} \mathrm{MG}$, the leaf discs from $\mathrm{L} 2$ retained significantly more chlorophyll than those from WT.

3.6 Overexpression of IbAKR upregulated the ROS scavenging-related genes

To investigate the impact of $I b A K R$-overexpression (IbAKR-OE) on the transcription of genes related to ROS scavenging, the expression levels of GPX, APX, MDHAR and $P O D$ under $\mathrm{Cd}$ stress were analyzed. The results showed that these genes were significantly upregulated (between 3- and 9-fold) in the transgenic lines compared with the WT after 2 weeks of treatment with $100 \mathrm{mmol} \cdot \mathrm{L}^{-1}$ $\mathrm{CdCl}_{2}$ (Fig. 6).

\section{Discussion}

AKRs encompass a large superfamily of $\mathrm{NAD}(\mathrm{P})(\mathrm{H})-$ dependent oxidoreductases ${ }^{[24]}$. In plants, a few AKRs have been reported to enhance tolerance to $\mathrm{Cd}$ stress ${ }^{[5,11]}$. In this 
(a)

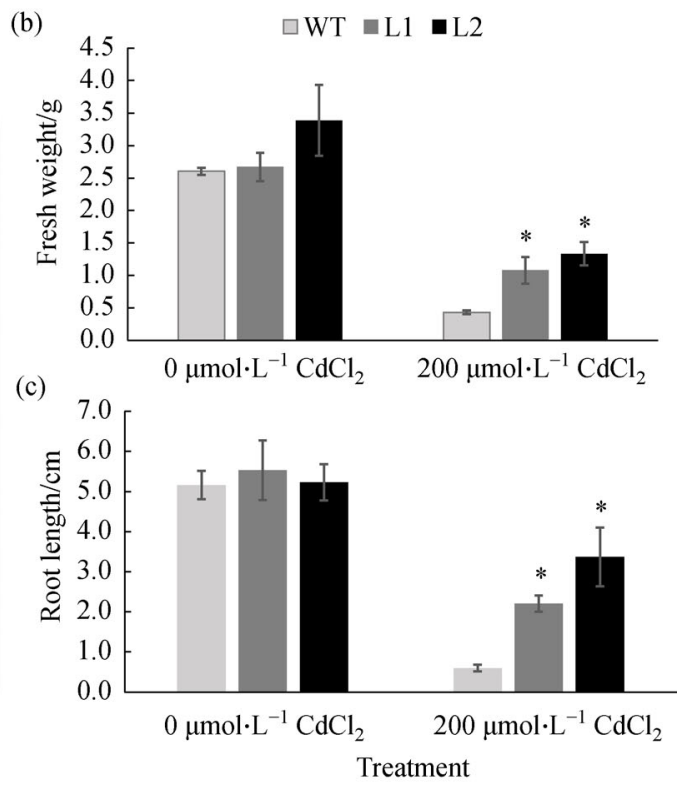

Fig. 3 Elevated cadmium tolerance in IbAKR-OE tobacco plants in the in vitro assay. (a) In vitro-grown tobacco plants were transferred to $1 / 2 \mathrm{MS}$ medium with or without $200 \mu \mathrm{mol} \cdot \mathrm{L}^{-1} \mathrm{CdCl}_{2}$. Photographs were taken 4 weeks after the start of treatment; (b, c) fresh weight (b) and root length (c) were measured. Data are presented as the mean $\pm \mathrm{SE}(n=3)$. ${ }^{*}$ and $* *$ indicate a significant difference from WT at $P<0.05$ and $<0.01$, respectively, by Student's $t$-test.

(a)

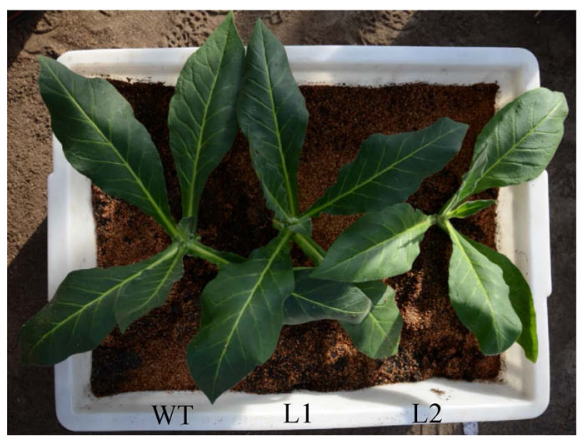

Before treatment

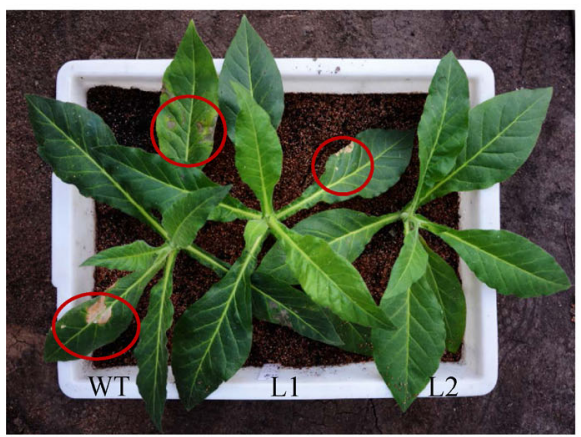

After treatment (b)

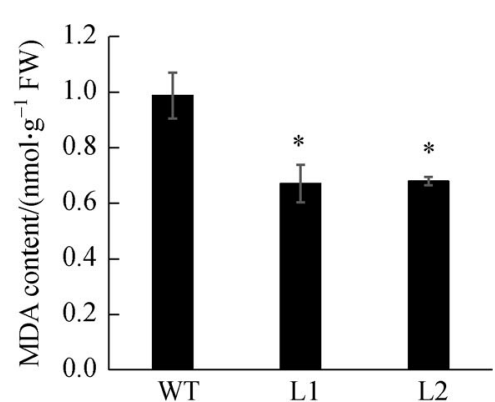

(d)

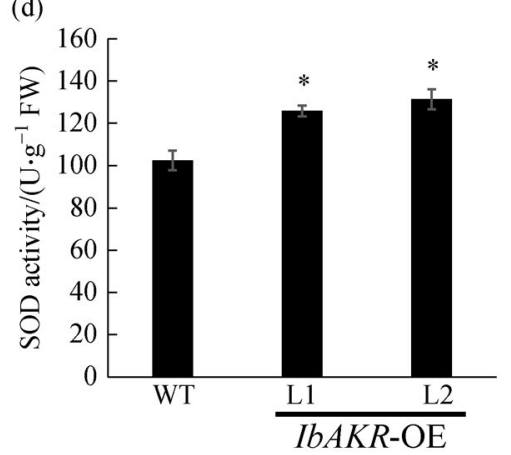

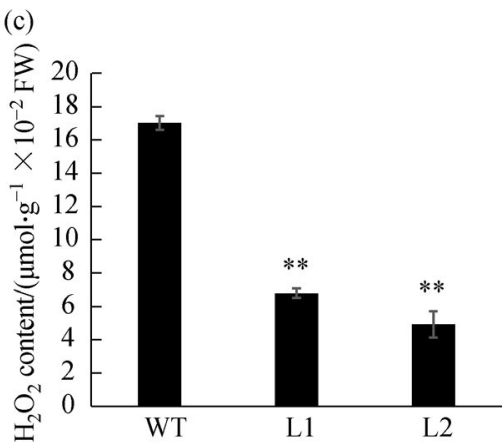

(e)

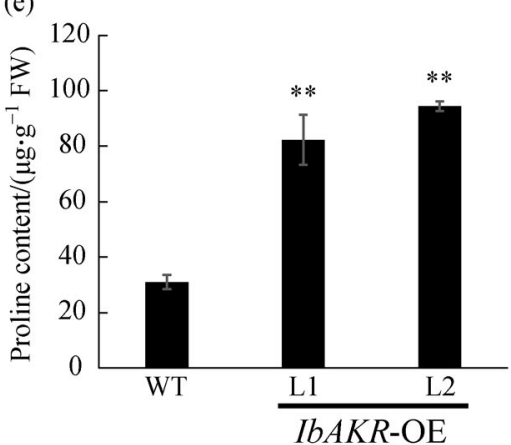

Fig. 4 Elevated cadmium tolerance in $I b A K R-\mathrm{OE}$ tobacco plants grown in pots. (a) Eight-week-old plants in a greenhouse were irrigated every $3 \mathrm{~d}$ with Hoagland solution containing $100 \mathrm{mmol} \cdot \mathrm{L}^{-1} \mathrm{CdCl}_{2}$ and morphological changes in the three lines (WT, L1, L2 from left to right) were photographed before and 2 weeks after the start of treatment; red circles indicate necrotic spots; (b-e) content of stress-related metabolites and superoxide dismutase (SOD) in transgenic lines and WT after treatment with $100 \mathrm{mmol} \cdot \mathrm{L}^{-1} \mathrm{CdCl}_{2}$ for 2 weeks. Data are presented as the mean $\pm \mathrm{SE}(n=3)$ * and ** indicate a significant difference from WT at $P<0.05$ and $<0.01$, respectively, by Student's $t$ test. 
(a)
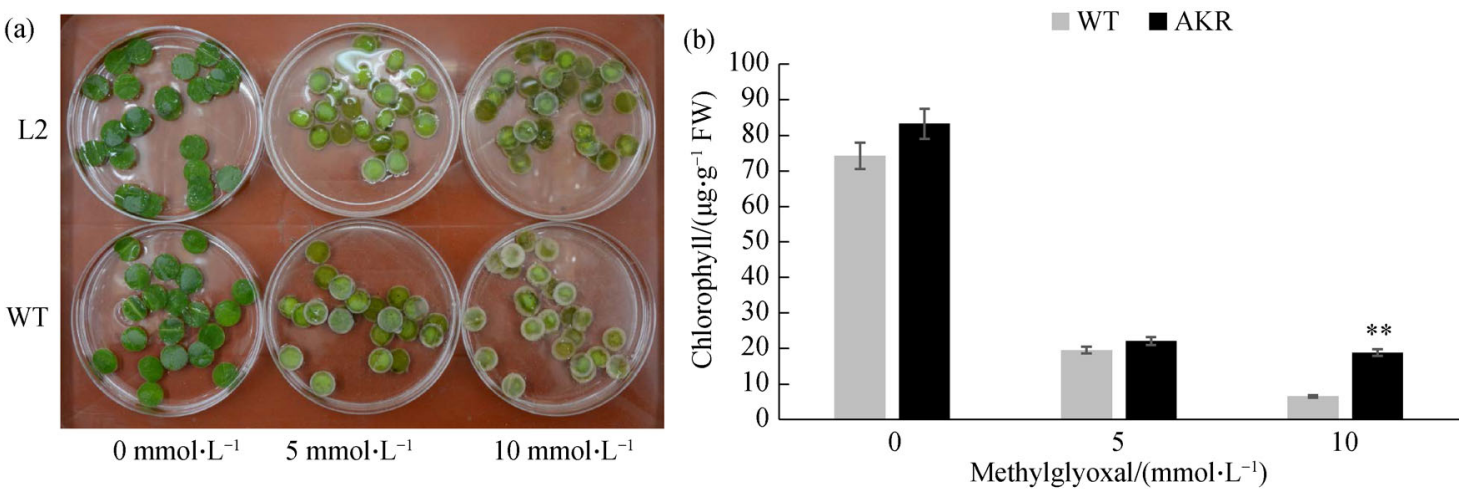

Fig. 5 Leaf disk senescence under methylglyoxal (MG) stress as measured by chlorophyll content. Chlorophyll content $\left(\mu \mathrm{g} \cdot \mathrm{g}^{-1} \mathrm{FW}\right)$ of leaf discs from WT and transgenic line L2 tobacco plants, kept for $3 \mathrm{~d}$ at different concentrations of MG. Data are presented as the mean $\pm \mathrm{SE}(n=3) .{ }^{*}$ and $* *$ indicate a significant difference from WT at $P<0.05$ and $<0.01$, respectively, by Student's $t$-test.
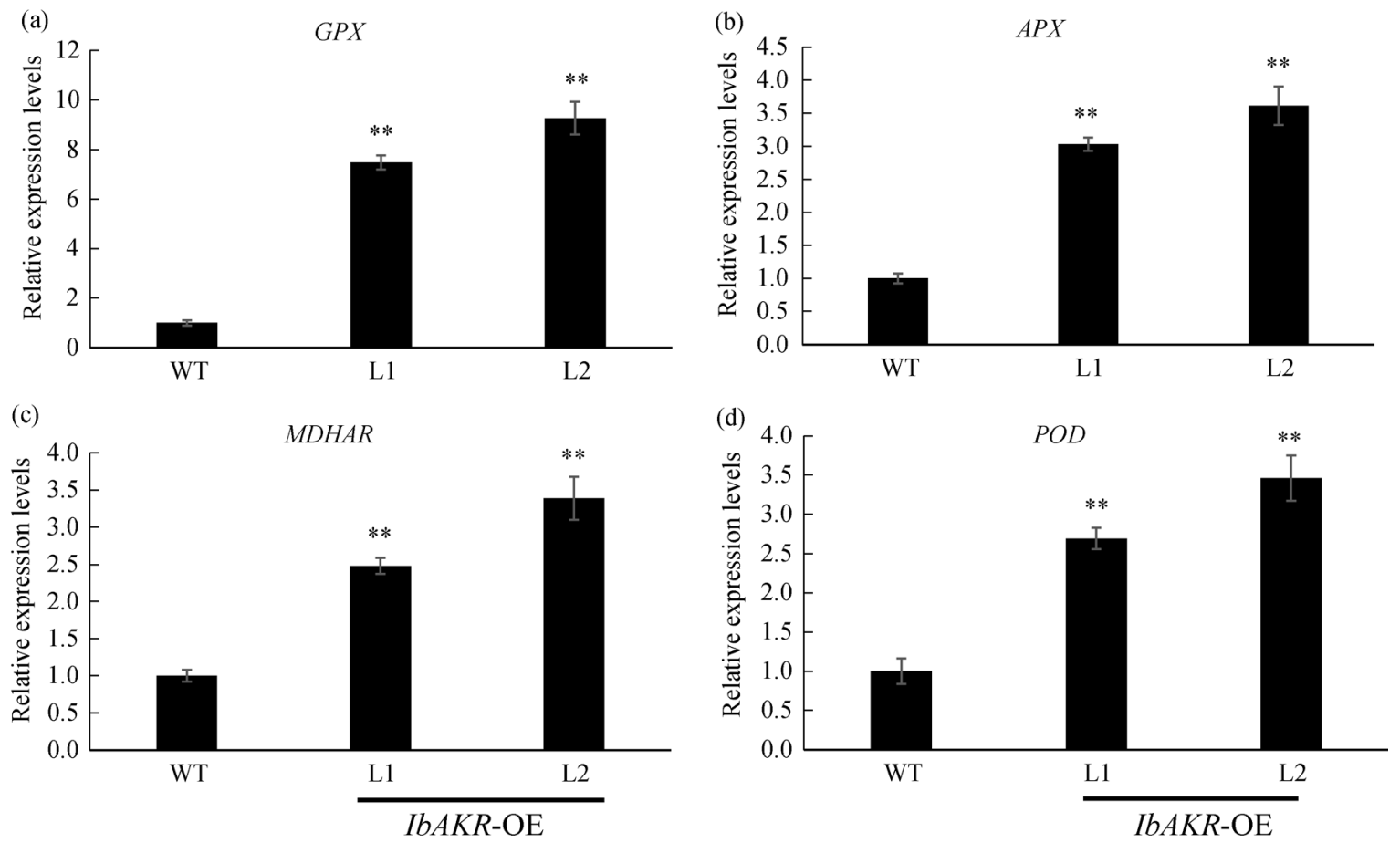

Fig. 6 Analysis of the expression of ROS scavenging-related genes in transgenic plants and WT. The relative expression levels of genes encoding guaiacol peroxidase $(G P X)$, ascorbate peroxidase $(A P X)$, monodehydroascorbate reductase $(M D H A R)$ and peroxidase $(P O D)$ were analyzed after treatment with $100 \mathrm{mmol} \cdot \mathrm{L}^{-1} \mathrm{CdCl}_{2}$ for 2 weeks. Data are presented as the mean $\pm \mathrm{SE}(n=3) . *$ and $* *$ indicate a significant difference from WT at $P<0.05$ and $<0.01$, respectively, by Student's $t$-test.

study, IbAKR was cloned from sweet potato. BLAST showed that IbAKR contained typical AKR signature motifs. According to the nomenclature of the AKR superfamily, IbAKR shared $51.63 \%$ ( $>40 \%$ ) amino acid identity with AKR13D1, suggesting that IbAKR belongs to family 13 of the AKR superfamily; however, its amino acid identity with the other members of family 13 was less than $60 \%$, suggesting that IbAKR is part of a new subfamily of family $13^{[26]}$. These results showed that IbAKR could be a novel member of the AKR superfamily.
The relative expression levels of $I b A K R$ in sweet potato increased 3- and 3.5-fold in response to $200 \mu \mathrm{mol} \cdot \mathrm{L}^{-1}$ $\mathrm{CdCl}_{2}$ and $10 \mathrm{mmol} \cdot \mathrm{L}^{-1} \mathrm{H}_{2} \mathrm{O}_{2}$, respectively, suggesting that IbAKR might have a role in Cd stress tolerance. The overexpression of $I b A K R$ enhanced tolerance to $\mathrm{Cd}$ stress in transgenic tobacco plants. Under Cd stress, transgenic plants showed better growth and rooting than WT (Fig. 3a-3c; Fig. 4a). This result indicates that the IbAKR gene may be used to improve plant tolerance to $\mathrm{Cd}$ stress. MDA is often considered a major lipid peroxidation 
marker, and its accumulation beyond a threshold level has been reported to cause irreparable damage to the photosynthetic machinery ${ }^{[27]}$. MG is a glycolysis-derived toxic aldehyde, and the accumulation of $\mathrm{MG}$ to a high concentration is toxic to cells, as it inhibits cell proliferation $^{[28]}$. In a previous study, OsAKR1 exhibited high catalytic activity in the reduction of toxic aldehydes, including lipid peroxidation-derived MDA and glycolysisderived MG, leading to an increased tolerance to oxidative and heat stress $^{[8]}$. In our study, we found that overexpressing $I b A K R$ could significantly reduce the levels of MDA relative to those in WT under Cd stress (Fig. 4b). Additionally, leaf discs from transgenic plants retained more chlorophyll after MG treatment (Fig. 5). These results suggest that $\mathrm{Cd}$ stress tolerance was enhanced in $I b A K R$ $\mathrm{OE}$ tobacco plants via scavenging MDA and MG and reduction in membrane damage.

In plants, Cd stress leads to the overproduction of ROS, which are highly reactive and toxic. ROS scavenging systems can detoxify ROS to minimize and/or prevent oxidative damage and cells respond to ROS by increasing the activity of ROS-scavenging enzymes ${ }^{[29]}$. As a key enzyme of the ROS scavenging system, SOD is usually induced by various abiotic stresses to promote the rapid dismutation of superoxide into oxygen and $\mathrm{H}_{2} \mathrm{O}_{2}$, which is subsequently removed by GPX, APX and POD through different pathways; moreover, MDHAR is needed to regenerate the active forms of antioxidants ${ }^{[30,31]}$. It has been reported that proline acts as a non-enzymatic ROS scavenger and can scavenge singlet oxygen and hydroxyl radicals ${ }^{[32]}$. In our study, IbAKR-OE tobacco plants had significantly higher SOD activity and proline levels and significantly lower $\mathrm{H}_{2} \mathrm{O}_{2}$ levels than WT (Fig. 4c-4e). qRT-PCR showed that the expression of GPX, $A P X$, $M D H A R$ and $P O D$ was significantly upregulated (Fig. 6), suggesting that the improved $\mathrm{Cd}$ stress tolerance of the transgenic tobacco plants is also due to enhanced ROS scavenging.

\section{Conclusions}

A novel aldo-keto reductase gene, I $b A K R$, has been successfully identified and cloned from the sweet potato cv. Lushu 3. The IbAKR-OE tobacco plants possessed higher tolerance than WT to Cd stress. This suggests the transgenic plants have a significant ability to scavenge MDA and MG. In addition, proline content and SOD activity were significantly higher and $\mathrm{H}_{2} \mathrm{O}_{2}$ levels were significantly lower in the transgenic plants than in the WT. Furthermore, ROS scavenging genes GPX, APX, MDHAR and $P O D$ were significantly upregulated in transgenic plants compared to the WT under Cd stress. Our results suggest that the overexpression of IbAKR enhanced $\mathrm{Cd}$ tolerance by reducing cytotoxic aldehydes and increasing the capacity for scavenging reactive oxygen species. This study indicates that the IbAKR gene may be useful for improving tolerance to $\mathrm{Cd}$ stress in plants.

Supplementary materials The online version of this article at https://doi. org/10.15302/J-FASE-2018225 contains supplementary materials (Table S1; Fig. S1).

Acknowledgements We thank Dr. Daniel Q. Tong, University of Maryland, USA, for English improvement. This work was supported by the National Natural Science Foundation of China (31271777) and the China Agriculture Research System (CARS-10, Sweet potato).

Compliance with ethics guidelines Jinxi Huo, Bing Du, Sifan Sun, Shaozhen He, Ning Zhao, Qingchang Liu, and Hong Zhai declare that they have no conflicts of interest or financial conflicts to disclose.

This article does not contain any studies with human or animal subjects performed by any of the authors.

\section{References}

1. Zhang X, Zhong T, Liu L, Ouyang X. Impact of soil heavy metal pollution on food safety in China. PLoS One, 2015, 10(8): e0135182

2. Gallego S M, Pena L B, Barcia R A, Azpilicueta C E, Iannone M F, Rosales E P, Zawoznik M S, Groppa M D, Benavides M P. Unravelling cadmium toxicity and tolerance in plants: insight into regulatory mechanisms. Environmental and Experimental Botany, 2012, 83(5): 33-46

3. Singh S, Parihar P, Singh R, Singh V P, Prasad S M. Heavy metal tolerance in plants: role of transcriptomics, proteomics, metabolomics, and ionomics. Frontiers in Plant Science, 2016, 6: 1143

4. Penning T M. The aldo-keto reductases (AKRs): overview. Chemico-Biological Interactions, 2015, 234: 236-246

5. Oberschall A, Deák M, Török K, Sass L, Vass I, Kovács I, Fehér A, Dudits D, Horváth G V. A novel aldose/aldehyde reductase protects transgenic plants against lipid peroxidation under chemical and drought stresses. Plant Journal, 2000, 24(4): 437-446

6. Hideg É, Nagy T, Oberschall A, Dudits D, Vass I. Detoxification function of aldose/aldehyde reductase during drought and ultraviolet-B (280-320 nm) stresses. Plant, Cell \& Environment, 2003, 26 (4): 513-522

7. Hegedüs A, Erdei S, Janda T, Tóth E, Horváth G, Dudits D. Transgenic tobacco plants overproducing alfalfa aldose/aldehyde reductase show higher tolerance to low temperature and cadmium stress. Plant Science, 2004, 166(5): 1329-1333

8. Turóczy Z, Kis P, Török K, Cserháti M, Lendvai A, Dudits D, Horváth G V. Overproduction of a rice aldo-keto reductase increases oxidative and heat stress tolerance by malondialdehyde and methylglyoxal detoxification. Plant Molecular Biology, 2011, 75 (4-5): 399-412

9. Vemanna R S, Vennapusa A R, Easwaran M, Chandrashekar B K, Rao H, Ghanti K, Sudhakar C, Mysore K S, Makarla U. Aldo-keto reductase enzymes detoxify glyphosate and improve herbicide resistance in plants. Plant Biotechnology Journal, 2017, 15(7): 794804

10. Kumar D, Singh P, Yusuf M A, Upadhyaya C P, Roy S D, Hohn T, Sarin N B. The Xerophyta viscosa aldose reductase (ALDRXV4) confers enhanced drought and salinity tolerance to transgenic 
tobacco plants by scavenging methylglyoxal and reducing the membrane damage. Molecular Biotechnology, 2013, 54(2): 292303

11. Éva C, Zelenyánszki H, Tömösközi-Farkas R, Tamás L. Overproduction of an Arabidopsis aldo-keto reductase increases barley tolerance to oxidative and cadmium stress by an in vivo reactive aldehyde detoxification. Plant Growth Regulation, 2014, 74(1): 5563

12. Éva C, Zelenyánszk H, Tömösközi-Farkas R, Tamás L. Transgenic barley expressing the Arabidopsis AKR4C9 aldo-keto reductase enzyme exhibits enhanced freezing tolerance and regenerative capacity. South African Journal of Botany, 2014, 93(93): 179-184

13. Kanayama Y, Mizutani R, Yaguchi S, Hojo A, Ikeda H, Nishiyama M, Kanahama K. Characterization of an uncharacterized aldo-keto reductase gene from peach and its role in abiotic stress tolerance. Phytochemistry, 2014, 104(3): 30-36

14. He W, Zhang L, Yi S, Tang X, Yuan Q, Guo M, Wu A, Qu B, Li H, Liao Y. An aldo-keto reductase is responsible for Fusarium toxindegrading activity in a soil Sphingomonas strain. Scientific Reports, 2017, 7(1): 1-13

15. Nisarga K N, Vemanna R S, Chandrashekar B K, Rao H, Vennapusa A R, Narasimaha A, Makarla U, Basavaiah M R. Aldoketoreductase 1 (AKRl) improves seed longevity in tobacco and rice by detoxifying reactive cytotoxic compounds generated during ageing. Rice, 2017, 10(1): 1-12

16. Wang Y, Li Y, Zhang H, Zhai H, Liu Q, He S. A soluble starch synthase I gene, IbSSI, alters the content, composition, granule size and structure of starch in transgenic sweet potato. Scientific Reports, 2017, 7(1): 2315

17. Cheng S, Huang C. Accumulation of cadmium uptake from soil in the edible root of root vegetables. Journal of Environmental Sciences, 2007, 17(2): 137-142

18. Schmittgen T D, Livak K J. Analyzing real-time PCR data by the comparative $C_{\mathrm{T}}$ method. Nature Protocols, 2008, 3(6): 1101-1108

19. Horsch R B, Fry J E, Hoffmann N L, Eichholtz D, Rogers S G, Fraley R T. A simple and general method for transferring genes into plants. Science, 1985, 227(4691): 1229-1231

20. Gao S, Yuan L, Zhai H, Liu C, He S, Liu Q. Transgenic sweet potato plants expressing an LOS5 gene are tolerant to salt stress. Plant Cell, Tissue and Organ Culture, 2011, 107(2): 205-213

21. He S, Han Y, Wang Y, Zhai H, Liu Q. In vitro selection and identification of sweet potato (Ipomoea batatas (L.) Lam.) plants tolerant to NaCl. Plant Cell, Tissue and Organ Culture, 2009, 96(1): 69-74

22. Alexieva V, Sergiev I, Mapelli S, Karanov E. The effect of drought and ultraviolet radiation on growth and stress markers in pea and wheat. Plant, Cell \& Environment, 2001, 24(12): 1337-1344

23. Porra R J, Thompson W A, Kriedemann P E. Determination of accurate extinction coefficients and simultaneous equations for assaying chlorophylls a and $\mathrm{b}$ extracted with four different solvents: verification of the concentration of chlorophyll standards by atomic absorption spectroscopy. BBA-Bioenergetics, 1989, 975(3): 384394

24. Agrawal C, Sen S, Yadav S, Rai S, Rai L C. A novel aldo-keto reductase (AKR17A1) of Anabaena sp. PCC 7120 degrades the rice field herbicide butachlor and confers tolerance to abiotic stresses in E. coli. PLoS One, 2015, 10(9): e0137744

25. Jez J M, Bennett M J, Schlegel B P, Lewis M, Penning T M. Comparative anatomy of the aldo-keto reductase superfamily. Biochemical Journal, 1997, 326(3): 625-636

26. Hyndman D, Bauman D R, Heredia V V, Penning T M. The aldoketo reductase superfamily homepage. Chemico-Biological Interactions, 2003, 143-144(2): 621-631

27. Ayala A, Muñoz M F, Argüelles S. Lipid peroxidation: production, metabolism, and signaling mechanisms of malondialdehyde and 4hydroxy-2-nonenal. Oxidative Medicine and Cellular Longevity, 2014, 2014(6): 360438

28. Ray S, Dutta S, Halder J, Ray M. Inhibition of electron flow through complex I of the mitochondrial respiratory chain of Ehrlich ascites carcinoma cells by methylglyoxal. Biochemical Journal, 1994, 303 (1): 69-72

29. Gill S S, Tuteja N. Reactive oxygen species and antioxidant machinery in abiotic stress tolerance in crop plants. Plant Physiology and Biochemistry, 2010, 48(12): 909-930

30. You J, Chan Z. ROS regulation during abiotic stress responses in crop plants. Frontiers in Plant Science, 2015, 6(12): 1092

31. Karuppanapandian T, Moon J C, Kim C, Manoharan K, Kim W. Reactive oxygen species in plants: their generation, signal transduction, and scavenging mechanisms. Australian Journal of Crop Science, 2011, 5(6): 709-725

32. Alia M P, Mohanty P, Matysik J. Effect of proline on the production of singlet oxygen. Amino Acids, 2001, 21(2): 195-200 